\title{
Testosterone Propionate
}

National Cancer Institute

\section{Source}

National Cancer Institute. Testosterone Propionate. NCI Thesaurus. Code C863.

A short acting oil-based injectable formulation of testosterone. Testosterone inhibits gonadotropin secretion from the pituitary gland and ablates estrogen production in the ovaries, thereby decreasing endogenous estrogen levels. In addition, this agent promotes the maintenance of male sex characteristics and is indicated for testosterone replacement in hypogonadal males. ( $\mathrm{NCl04)}$ 